\title{
Amplification and Detection of Transposon Insertion Flanking Sequences Using Fluorescent $M u$ AFLP
}

BioTechniques 32:1090-1097 (May 2002)

\section{Edwards, J. Coghill, J. Batley, M. Holdsworth, and K.J. Edwards \\ University of Bristol, Bristol, UK}

\section{INTRODUCTION}

Transposon insertion mutagenesis is a powerful tool for modifying gene expression and understanding gene function (10). Recent developments allow ing the amplification of insertion flanking sequences has led to improved methods for the efficient molecular characterization of transposon insertion events (2). This is now the basis of several methods for gene knockout identification and cloning, including the direct sequencing of amplified transposon flanking regions (3) and hybridizationbased gene insertion detection (8). Previous work has shown that Mutator-amplified fragment length polymorphism (MuAFLP) is an efficient and reproducible technique for amplifying Mutator $(M u)$ transposon flanking sequences from individual $M u$-active maize plants (3). Amplified $M u$ flanking sequences have previously been visualized by agarose gel electrophoresis, followed by staining with ethidium bromide or by PAGE fractionation of radiolabeled fragments, followed by autoradiography. These methods are limited by low resolution and poor quantification of the products, along with difficulties in accurately sizing the amplified products. To overcome these limitations and permit MuAFLP optimization, we have developed a technique to allow the accurate, automated quantification and sizing of MuAFLP products. The incorporation of a fluorescently labeled oligonucleotide primer during amplification enables the detection and quantification of $M u$-specific amplified products using an automated genotyper such as an ABI
PRISM $^{\circledR} 377$ DNA sequencer (Applied Biosystems, Foster City, CA, USA). The addition of fluorescently labeled molecular size markers further allows the accurate sizing of the amplified products. This permits the direct comparison of samples fractionated on different PAGE gels. Using this detection method, we have assessed parameters affecting $M u$ AFLP toward optimizing the amplification of $M u$ flanking sequences. We have also assessed the suitability of this method for the amplification of $M u$ flanking sequences from increasingly complex templates prepared from increasing numbers of pooled $M u$-active plants. Because PCR amplification of insertion flanking sequences is important for the characterization of both transposon and transgenic insertion mutagenesis in a wide variety of organisms, we suggest that the development of automated fluorescent methods for amplified insertion flanking sequence detection may greatly enhance the characterization of insertion events for a range of mutagenesis systems.

\section{MATERIALS AND METHODS}

\section{Growth and Maintenance of Maize Plants}

The $M u$-active maize seed in a W22 background was a kind gift of Dr. Martienssen of the Cold Spring Harbor Laboratory. Plants were grown as described previously (6). $M u$-active plants were grown as heterozygote families by random mating within the $M u$-active pool. The Southern blot hybridization 
(7) of the DNA prepared from this material indicated that individual plants contained between 35 and $50 \mathrm{Mu}$ elements, when probed with a [32P]ATPlabeled $M u$-inverted repeat.

\section{Amplification and Detection of $M u$ Flanking Sequences}

$M u$ insertion flanking sequences were isolated by a modification of the method of Frey et al. (2). Total genomic DNA (100 ng) was digested with $4 \mathrm{U}$ $M l u \mathrm{I}$ and $4 \mathrm{U} M s e \mathrm{I}$ restriction enzymes in a volume of $30 \mu \mathrm{L}$ for $1 \mathrm{~h}$ at $37^{\circ} \mathrm{C}$. Following restriction digestion, $100 \mathrm{ng}$ biotinylated $M l u \mathrm{I}$ adapter (consisting of a biotinylated 17-mer, biotin-5'-CTCG TAGACTGCGTAAC-3' and a complementary 15-mer, 5'-CGCGGTTACG CAGTC-3'), 100 ng nonbiotinylated Mse I adapter (consisting of a 16 mer, 5' GACGATGAGTCCTGAG-3' and a complementary 14-mer, 5'-TACTCA GGACTCAT-3') and $1 \mathrm{mM}$ ATP were added to the digestion mixture along with $2.5 \mathrm{U}$ T4 DNA ligase to make a total volume of $50 \mu \mathrm{L}$. The ligation was allowed to proceed for $15 \mathrm{~h}$ at $37^{\circ} \mathrm{C}$. Genomic fragments linked to the $M l u \mathrm{I}$ adapters were isolated and purified using $10 \mu \mathrm{L}$ magnetic streptavadin beads (Dynal, Lake Success, NY, USA) (9). Following purification, a $1-\mu \mathrm{L}$ aliquot of the DNA-beads complex was subjected to amplification using a fluorescently labeled primer specific for a conserved region of the $M u$-inverted repeat MuB (5'-TETCTTCGTCCATAATGG CAATTATCTC-3'), MuA (5'-HEXTCGAATCCCCTTCTCTCTTCGTCC-3'), or MuC (5'-FAM GCCTCCATTTCGTCGAATCCC-3'), and a primer specific for the $M s e I$ adapter (5'GACGATGAGTCCTGAGTAA-3') in $25 \mu \mathrm{L}$ with $1 \mathrm{U}$ AmpliTaq Gold ${ }^{\circledR}$ DNA polymerase (Applied Biosystems) using standard cycling conditions of $94^{\circ} \mathrm{C}$ for $10 \mathrm{~min}$, followed by 35 cycles of $94^{\circ} \mathrm{C}$ for $1 \mathrm{~min}, 55^{\circ} \mathrm{C}$ for $1 \mathrm{~min}$, and $72^{\circ} \mathrm{C}$ for $3 \mathrm{~min}$, with a final extension time of 10 min at $72^{\circ} \mathrm{C}$, except where stated in the text. Following amplification, a $1-\mu \mathrm{L}$ aliquot of the fragments was fractionated and detected using an ABI PRISM 377 automated sequencer with internal TAMRA 2500 size standards (Applied Biosystems). DNA fragments were characterized using GeneScan ${ }^{\circledR}$ and Genotyper $^{\circledR}$ (Applied Biosystems) software. Fragments were automatically scored for size and peak height, and the resulting data were used to produce histograms for visual assessment. Each amplification was repeated between 4 and 14 times to assess reproducibility.

\section{RESULTS}

\section{Reproducibility of $M u A F L P$}

Products from 14 independent am plifications were compared to assess the reproducibility of amplification efficiency and profile of amplification products. Total amplified product from each reaction, measured as the sum of product fluorescent intensities, ranged from 17516 to 39646 , with a mean of 25923 and a CV of $38.8 \%$. The uncorrected $\mathrm{CV}$ for individual products ranged between $15.5 \%$ and $51.1 \%$. When products were normalized to total amplified product, the $\mathrm{CV}$ ranged between $12.6 \%$ and $39.8 \%$, with a mean CV of $24.9 \%$.

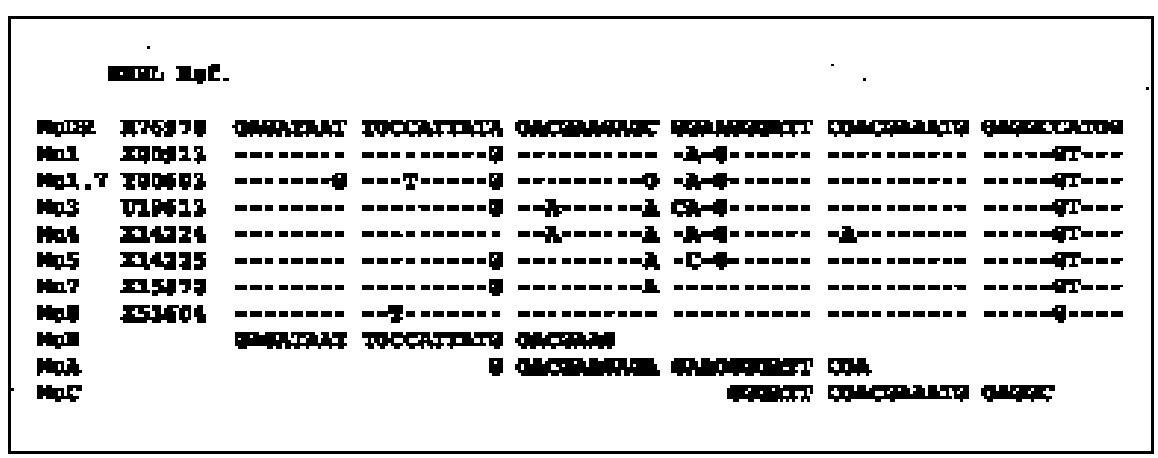

Figure 1. Alignment of $M u$ terminal sequences and $M u$-specific oligonucleotide primers. Maize $M u$ specific sequences were identified and retrieved from the EMBL database. Conserved terminal sequences were aligned and used to design the $\mathrm{Mu}$-specific oligonucleotide primers $\mathrm{MuA}, \mathrm{MuB}$, and $\mathrm{MuC}$.

\section{Optimization of the MuAFLP Technique}

Primer design. Three nested oligonucleotide primers designed to the conserved region of the $M u$ terminal inverted repeat (TIR) (Figure 1) were tested for their ability to reproducibly amplify $M u$ insertion flanking sequences from an individual plant. The results are summarized in Table 1. A total of 30 products were amplified using either MuA or MuB TIR-specific primers in conjunction with a MseI-specific adapter primer. Each MuB fragment corresponded to a MuA fragment $16 \pm$ 1 bases greater in size. Amplification using the $\mathrm{MuC}$ primer identified 24 of the 30 fragments, nested $12 \pm 1 \mathrm{bp}$ and $28 \pm 1 \mathrm{bp}$, respectively, with a corresponding MuA and MuB product. For the remaining six products amplified using $\mathrm{MuA}$ and $\mathrm{MuB}$, no corresponding $\mathrm{MuC}$ product was detected.

PCR conditions. PCR annealing temperatures and extension times were independently assessed for their ability to amplify reproducibly $M u$ AFLP products. In each case, amplification was performed using identical template, primer, and reaction conditions, with the only variation being the amplification parameter under scrutiny. Analysis of $M u$ AFLP products amplified using annealing temperatures between $50^{\circ} \mathrm{C}$ and $53^{\circ} \mathrm{C}$ revealed only a minor variation in $M u$ AFLP profile and the amplification of an additional product of 359 bp at $53^{\circ} \mathrm{C}$. However, annealing temperatures of $45^{\circ} \mathrm{C}, 55^{\circ} \mathrm{C}$, and $60^{\circ} \mathrm{C}$ resulted in a reduced number of amplified fragments, along with a significant reduction in total amplified product (Figure $2)$. No amplification products were detected at an annealing temperature of $65^{\circ} \mathrm{C}$ (data not shown). Examining MuAFLP products amplified using extension times of between $30 \mathrm{~s}$ and $3 \mathrm{~min}$ showed an increase in the relative fluorescent intensity of the longer fragments (>500 bp), with an increase in extension time. Novel long fragments were also observed when increased extension times were used (data not shown).

Template complexity. The efficiency of $M u$ flanking sequence amplification from increasingly complex templates was assessed using fluorescent $\mathrm{Mu}$ AFLP. $M u$ flanking sequences were am 
plified from five individual $M u$-active plants and from DNA pooled from either two or all five plants (Figure 3). $M u$ AFLP performed on individual plants identified between 27 and $47 M u$ flanking sequences. Fifty-six $M u$ flanking sequences were amplified from DNA pooled from two plants with products representative of $M u$ flanking sequences amplified from the two plants independently. However, $M u$ flanking sequences amplified from DNA pooled from five plants were not representative of fragments amplified from the five plants individually, and the number of amplified $M u$ fragments was not observed to increase in complex template mixtures derived from more than two plants.

\section{DISCUSSION}

\section{General Outline of the Fluorescent MuAFLP Technique}

DNA sequences flanking $M u$ transposon insertions are amplified, follow ing a modified adapter ligation and biotin-linked magnetic selection method (2). Genomic DNA is first extracted and digested with two restriction endonucleases, $M s e \mathrm{I}$ and $M l u \mathrm{I} . M l u \mathrm{I}$ is a methylation-sensitive restriction endonuclease with a six-base target sequence that cuts rarely in the maize genome, though within the terminal repeats of common $M u$ elements. MseI has a four-base target sequence and cuts frequently in the maize genome. The majority of fragments produced from this double digestion are $\mathrm{Mse}$ $M s e I$, with fewer $M l u \mathrm{I}-M s e \mathrm{I}$ fragments. Ligation of $M s e \mathrm{I}$ and biotinylated $M l u \mathrm{I}$ adapters allows the enrichment of rare MluI-MseI fragments with streptavidinlinked magnetic beads. This pool of $M l u I-M s e$ I fragments is used as a template for PCR amplification of $M u$ flanking sequences using a fluorescently labeled $M u$-specific primer and a MseI adapter-specific primer. Amplified $M u$ flanking sequences are fractionated and detected on an automated sequencer such as an ABI PRISM 377 using internal fluorescently labeled molecular size standards. The fragments are quantified and their size is calculated using GeneScan, Genotyper, and Genographer software (freely

Table 1. Fluorescent $M u$ AFLP Products Amplified Using Three Nested $M u$-Specific Primers

\begin{tabular}{|c|c|c|c|}
\hline $\begin{array}{l}\text { Amplified } M u \\
\quad \text { flanking } \\
\text { sequence no. }\end{array}$ & $\begin{array}{l}\text { Fragment size } \\
\text { (bp) amplified } \\
\text { using primer MuB }\end{array}$ & $\begin{array}{l}\text { Fragment size } \\
\text { (bp) amplified } \\
\text { using primer MuA }\end{array}$ & $\begin{array}{l}\text { Fragment size } \\
\text { (bp) amplified } \\
\text { using primer MuC }\end{array}$ \\
\hline 1 & 96 & 112 & 124 \\
\hline 2 & 109 & 125 & Not Detected \\
\hline 3 & 124 & 140 & 152 \\
\hline 4 & 133 & 149 & 161 \\
\hline 5 & 137 & 153 & 165 \\
\hline 6 & 158 & 174 & 186 \\
\hline 7 & 173 & 189 & Not Detected \\
\hline 8 & 197 & 213 & 225 \\
\hline 9 & 203 & 219 & Not Detected \\
\hline 10 & 207 & 223 & 235 \\
\hline 11 & 216 & 232 & Not Detected \\
\hline 12 & 225 & 240 & 251 \\
\hline 13 & 253 & 269 & 281 \\
\hline 14 & 310 & 326 & 338 \\
\hline 15 & 318 & 333 & 345 \\
\hline 16 & 345 & 361 & 372 \\
\hline 17 & 355 & 370 & Not Detected \\
\hline 18 & 366 & 381 & 393 \\
\hline 19 & 377 & 393 & 405 \\
\hline 20 & 418 & 435 & 442 \\
\hline 21 & 427 & 443 & Not Detected \\
\hline 22 & 463 & 481 & 494 \\
\hline 23 & 511 & 526 & 537 \\
\hline 24 & 538 & 554 & 566 \\
\hline 25 & 571 & 587 & 598 \\
\hline 26 & 598 & 614 & 626 \\
\hline 27 & 636 & 653 & 665 \\
\hline 28 & 666 & 683 & 694 \\
\hline 29 & 684 & 700 & 714 \\
\hline 30 & 726 & 742 & 754 \\
\hline
\end{tabular}

available from http://hordeum.oscs. montana.edu/genographer).

\section{Optimization of the MuAFLP Technique}

Reproducibility of fluorescent Mu AFLP. Fluorescent MuAFLP was assessed for both reproducibility of product profile and total amplified product abundance, measured as the sum of fluorescent intensities for each identified product. Among 14 repeated ampli- fications, product abundance varied by $38.8 \%$. This variation may reflect the efficiency of amplification and/or experimental variation such as gel loading. While the uncorrected $\mathrm{CV}$ for individual products ranged between $15.5 \%$ and $51.1 \%$, a proportion of this variation is due to differences in total product abundance rather than product profile. When products were normalized to total amplified product, the $\mathrm{CV}$ ranged between $12.6 \%$ and $39.8 \%$, with a mean $\mathrm{CV}$ of $24.9 \%$. This variation in product profile 


\section{Research Report}

reflects the complex nature of multiplex PCR from a pool of variant templates.

Primer design. The $M u$ family of transposable elements shares conserved TIRs (Figure 1). However, variation in the sequence of these TIRs among fam ily members may lead to selective am plification of insertion flanking sequences in a primer-dependent manner. Three nested $M u$-specific primers, $\mathrm{MuA}, \mathrm{MuB}$, and MuC (Figure 1), were tested for their ability to reproducibly amplify $M u$ flanking sequences from individual maize plants. A total of 30 fragments were amplified from the DNA of a single plant using either $\mathrm{MuA}$ or MuB. Each of the $30 \mathrm{MuA}$ fragments were $16 \pm 1 \mathrm{bp}$ longer than a corresponding MuB-amplified fragment, indicating that these products represent true nested amplified $M u$ flanking se-

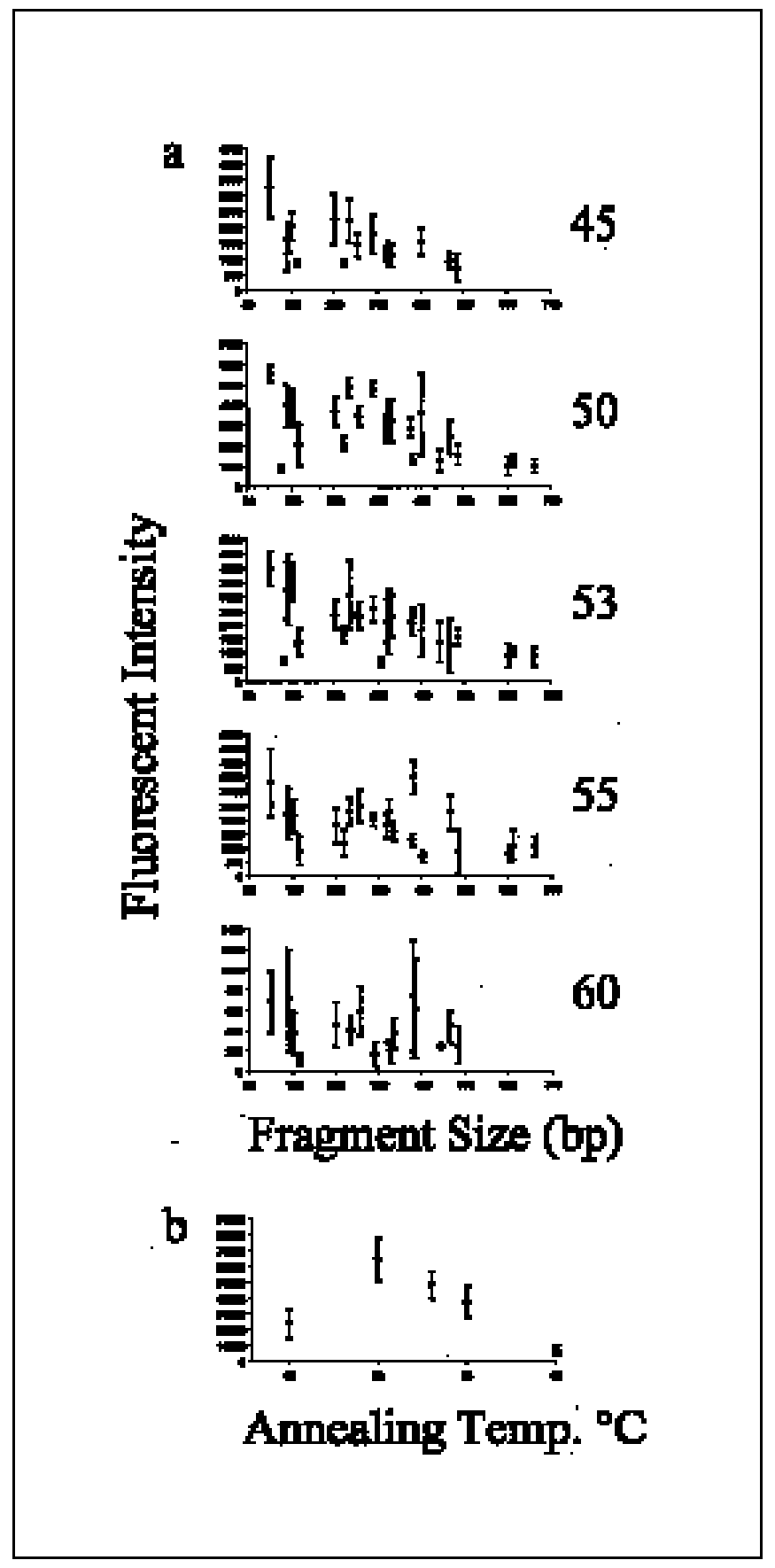

Figure 2. Effect of annealing temperature on $M u$ AFLP. $M u$ transposon flanking sequences were amplified from an individual $M u$-active maize plant by fluorescent $M u A F L P$ using annealing temperatures of $45^{\circ} \mathrm{C}, 50^{\circ} \mathrm{C}, 53^{\circ} \mathrm{C}$, $55^{\circ} \mathrm{C}$, and $60^{\circ} \mathrm{C}$. Each amplification was repeated four times to determine the mean fluorescent intensity, and error bars denote one standard deviation.

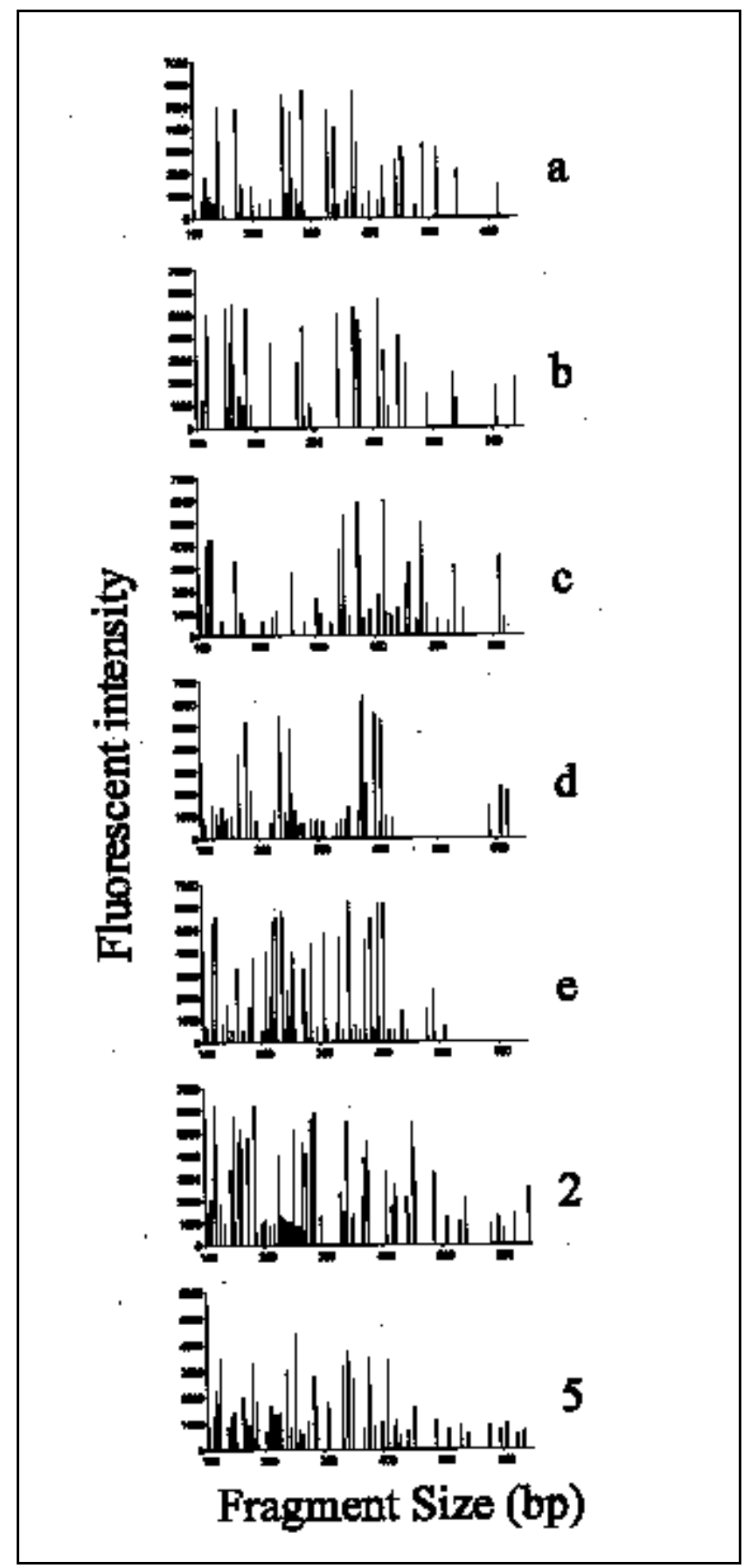

Figure 3. Effect of template complexity on MuAFLP. $M u$ transposon flanking sequences were amplified by fluorescent $M u$ AFLP from individual plants a-e or increasingly complex templates representing a pool of two plants $(\mathrm{a}+\mathrm{b})$ or five plants $(\mathrm{a}-\mathrm{e})$. 
quences. Amplification using the $\mathrm{MuC}$ primer identified 24 of the $30 \mathrm{Mu}$ flanking sequences identified using MuA or MuB primers, differing in size by $12 \pm 1$ and $28 \pm 1 \mathrm{bp}$, respectively. Amplification of these 24 flanking sequences was observed to be efficient and reproducible using the MuC primer, indicating that the failure of MuC to amplify the remaining six products is due to sequence variation within the $\mathrm{MuC}$ binding region of these $M u$ TIRs. Alignment of the few sequenced $M u$ TIRs identified in the public databases (Figure 1) suggests that the $\mathrm{MuC}$ primer binding site is highly conserved; however, the TIR sequences of many $M u$ and $M u$-like elements have yet to be determined $(1,5)$. Future sequencing of further $M u$ TIRs may confirm a greater variation within the MuC-binding region.

PCR conditions. Amplification of $M u$ flanking sequences using $M u$ AFLP is complex because of the large number of target sequences and the potential sequence variability in the $M u$ TIR region. To optimize the amplification of these sequences, a range of PCR annealing temperatures and extension times were assessed, with the amplification products in each case analyzed by fluorescent MuAFLP. The annealing temperature of the PCR cycle is critical for the efficient amplification of PCR products. While there are numerous methods for the calculation of annealing temperature between a specific oligonucleotide and template DNA, their accuracy is limited. Therefore, the experimental optimization of PCR annealing temperature is required. Using fluorescent $M u$ AFLP, amplification was observed to be most efficient at $50^{\circ} \mathrm{C}$ and $53^{\circ} \mathrm{C}$, with only a minor variation in product profile between these temperatures. Poor amplification was observed at annealing temperatures of $45^{\circ} \mathrm{C}$ and $60^{\circ} \mathrm{C}$, with intermediate am plification at $55^{\circ} \mathrm{C}$ (Figure 2). Quantification of size fractionated fluorescent $M u$ AFLP products amplified using extension times ranging from $30 \mathrm{~s}$ to 3 min identified a requirement for extension times of up to $3 \mathrm{~min}$ for the efficient amplification of large fragments (>500 bp). This reflects the efficiency of the Taq DNA polymerase in extending the full length of each of these longer products. Extension times of 1 min are normally sufficient for the amplification of products of this size (up to $1 \mathrm{kbp}$ ) (4). The longer extension time required to fully extend $M u$ AFLP products may reflect the large number of fragments being amplified or be due to the presence of inhibitory DNA secondary structures at the $M u$ TIRs.

Template complexity. We have shown that the MuAFLP technique is ef- 
ficient and reproducible when amplifying $M u$ flanking sequences from individual plants. Given the trend toward high-throughput functional genomics in maize, the simultaneous amplification of $M u$ flanking sequences representing a pool of $M u$-active maize plants would aid high-throughput identification and characterization of gene insertions. We have applied fluorescent $M u$ AFLP to amplify $M u$ flanking sequences from increasing numbers of pooled $M u$-active plants, with the aim of identifying the maximum number of $M u$ flanking sequences that may be amplified in a single $M u$ AFLP reaction. Reproducible and representative amplification of 56 products was observed using a pool of two plants. However, products amplified from a pool of five or more plants were not representative of the five individual plants making up the pool. This limitation in the number of $M u$ fragments am plified in each reaction reflects the com plexity associated with the simultaneous amplification from a large number of heterogeneous templates. Abundance of any particular product in the amplified reaction is dependent on several template-related factors: base-pair mismatches between the $M u$ template and $M u$ oligonucleotide primer may decrease its final abundance, longer tem plates may be amplified less efficiently than shorter templates, and in the case of pooled DNA from related $M u$-active maize plants, parental insertions present in two or more pool samples are overrepresented in the template mixture and thus may be more abundant in the am plified product. Where $M u$ flanking sequences are amplified from individual plants, template number is low and am plification is both efficient and reproducible. Here we have demonstrated that when DNA from several plants is pooled, the large numbers of templates competing for amplification lead to a reduction in amplification efficiency and poor representation of the initial $M u$ flanking fragments.

\section{CONCLUSIONS}

We have developed a fluorescent $M u$ AFLP technique for the quantification and size determination of amplified $M u$ transposon flanking sequences.
This has enabled the empirical optimization of PCR conditions for MuAFLP and the assessment of different $M u$-specific primers. Analysis of $M u$ flanking sequences amplified from complex templates representing multiple $M u$-active plants indicates a reduced efficiency of $M u$ flanking sequence amplification from pools of more than two plants. This optimized procedure has applications in the study of $M u$ transposition and inheritance in maize and may be further modified for the detection of sequences flanking transposon or transgene insertions in a wide range of organisms.

\section{ACKNOWLEDGMENTS}

IACR-Long Ashton receives grantaided support from the Biotechnology and Biological Sciences Research Council of the UK. D. Edwards and J. Coghill were funded by the BBSRC Investigating Gene Function initiative (Cereal IGF programme, grant no. 233/IGF1240).

\section{REFERENCES}

1.Benito, M.I. and V. Walbot. 1997. Characterization of the maize Mutator transposable element MURA transposase as a DNA-binding protein Mol. Cell. Biol. 7:5165-5175.

2.Frey, M., C. Stettner, and A. Gierl. 1998. A general method for gene isolation in tagging approaches: amplification of insertion mutagenized sites (AIMS). Plant J. 13:717-721.

3.Hanley, S., D. Edwards, D. Stevenson, S. Haines, M. Hegarty, W. Schuch, and K.J. Edwards. 2000. Identification of transposon tagged genes by the random sequencing of Mutator-tagged DNA fragments from Zea mays. Plant J. 22:557-566.

4.Innis, M.A., K.B. Myambo, D.H. Gelfand, and M.A.D. Brow. 1988. DNA sequencing with Thermus-aquaticus DNA-polymerase and direct sequencing of polymerase chain reaction-amplified DNA. Proc. Natl. Acad. Sci. USA 85:9436-9440.

5.Lisch, D.R., M. Freeling, R.J. Langham, and M.Y. Choy. 2001. Mutator transposase is widespread in the grasses. Plant Physiol. 125:1293-1303.

6.Neuffer, M.G. 1993. Growing maize for genetic studies, p. 197-208. In M. Freeling and V. Walbot (Eds.), The Maize Handbook. Springer Verlag, New York.

7.Southern, E.M. 1975. Detection of specific sequences among DNA fragments separated by gel electrophoresis. J. Mol. Biol. 98:503-517.

8. Tissier, A.F., S. Marillonnet, V. Klimyuk, K. Patel, M.A. Torres, G. Murphy, and J.D.G. Jones. 1999. Multiple independent defective
Suppressor-Mutator transposon insertions in Arabidopsis: a tool for functional genomics. Plant Cell 11:1841-1852.

9.Tong, X.C. and L.M. Smith. 1992. Solid-phase method for the purification of DNA sequencing reactions. Anal. Chem. 64:2672-2677.

10. Walbot, V. 1992. Strategies for mutagenesis and gene cloning using transposon tagging and T-DNA insertional mutagenesis. Annu. Rev. Plant Physiol. Plant Mol. Biol. 43:49-82.

Received 18 June 2001; accepted 4 December 2001.

Address correspondence to:

Dr. David Edwards

Crop Genetics

IACR-Long Ashton Research Station Department of Agricultural Sciences

University of Bristol

Long Ashton, Bristol BS41 9AF, UK

e-mail:dave.edwards@bbsrc.ac.uk

For reprints of this or any other article, contact Reprints@BioTechniques.com 